\title{
RF-Activated Standing Surface Acoustic Wave for On-Chip Particle Manipulation
}

\author{
Jinhong Guo, Member, IEEE, Joshua L. W. Li, Fellow, IEEE, Yu Chen, \\ Leslie Y. Yeo, James R. Friend, and Yuejun Kang
}

\begin{abstract}
On-chip flow cytometry provides a powerful tool to characterize cell samples for point-of-care diagnosis. In particular, sample focusing at specific locations along the microchannel is crucial to ensure the accuracy of detection. In this paper, we present a simple strategy of interfacing an RF-activated standing surface acoustic wave (SSAW) substrate with a microfluidic channel, and use this device to study the dynamic process of particle aggregation along the microchannel. Specifically, the SSAW generated by two parallel interdigital transducers induces an acoustic radiation force that propels particles suspended in the liquid toward the pressure nodes whose locations are tunable by judicious choice of the applied SSAW frequency. We also carry out a theoretical analysis that provides an estimation of the time for the particle assembly, which is validated by experimental results. This SSAW transducer can therefore be easily integrated into a microfluidic chip with moderate energy consumption, offering a convenient and effective solution in the development of on-chip flow cytometry.
\end{abstract}

Index Terms-Acoustic, acoustic wave components, RF/microwaves, surface acoustic wave (SAW) measurement, SAW devices.

\section{INTRODUCTION}

$\mathbf{T}$ HE DETECTION and characterization of biological particles, such as cells and biomolecules, is a fundamental technique in biology and medical biotechnology. Since

Manuscript received March 21, 2014; revised May 21, 2014 and July 16, 2014; accepted July 18, 2014. Date of publication August 01, 2014; date of current version September 02, 2014. The work of Y. Kang was supported by the Ministry of Education of Singapore (RG 26/11) under a Tier-1 Academic Research Fund. This paper is an expanded version from the IEEE MTT-S International Microwave Workshop Series on RF and Wireless Technologies for Biomedical and Healthcare Applications, Singapore, Dec. 9-11, 2013.

J. Guo and Y. Kang are with the School of Chemical and Biomedical Engineering, Nanyang Technological University, Singapore 637459 (e-mail: jguo002@e.ntu.edu.sg; yuejun.kang@ntu.edu.sg).

$\mathrm{J} . \mathrm{L} . \mathrm{W} . \mathrm{Li}$ is with the Institute of Electromagnetics, University of Electronic Science and Technology of China, Sichuan 611731, China (e-mail: 1wli@ieee. org).

Y. Chen is with the A*STAR Institute of Microelectronics, Singapore 117685 (e-mail: cheny1@ime.a-star.edu.sg).

L. Y. Yeo and J. R. Friend are with the School of Civil, Environmental and Chemical Engineering and the School of Electrical and Computer Engineering, RMIT University, Melbourne, Vic. 3000, Australia (e-mail: leslie.yeo@rmit. edu.au; james.friend@rmit.edu.au).

Color versions of one or more of the figures in this paper are available online at http://ieeexplore.ieee.org.

This paper has supplementary downloadable multimedia material available at http://ieeexplore.ieee.org provided by the authors. This includes a video of standing surface acoustic waves in a microfluidic channel measured by a microscanning Doppler vibrometer. This video is $0.5 \mathrm{MB}$ in size. Windows Media Player or QuickTime required for viewing.

Digital Object Identifier 10.1109/TMTT.2014.2342667 bioparticles usually exist in fluidic environment, microfluidics-based lab-on-a-chip devices provide excellent platforms for various biomedical manipulations and assays. The amazingly fast development of lab-on-a-chip technology in recent decades has had a profound impact on the food and healthcare industries [1]-[3], as well as novel applications in bio-defense against bioterrorism and bio-warfare [4]. Many experimental techniques for microparticle manipulation have been extensively reported in prior studies, such as using biochemical [5], electrokinetic [6], optical [7], and magnetic [8] methods. Compared to these conventional techniques, another popular method that utilizes acoustics to drive microfluidic actuation has shown distinct advantage as an easy tool for manipulation of colloidal particles [9]-[11]. Using various types of ultrasonic transducers, acoustic energy can be easily transmitted into colloidal particle suspensions in confined micro-geometry. The acoustic radiation force due to the standing wave in the carrier medium drives the particles to the local pressure nodes. This unique phenomenon can be applied for particle concentration, positioning, and fractionation. Compared with other popular techniques, acoustic particle manipulation does not require fluorescence or magnetic labels; avoids direct coupling of the electric field into the fluid, therefore circumventing undesirable electrochemical reaction and joule heating effects; and does not affect the bioelectricity, and thus causes less stress on the biological cell membrane. Consequently, acoustic methods have higher biocompatibility for biomedical applications. A typical and interesting application is to use acoustic focusing of biological cells into a thin stream for sample preparation and for micro flow cytometry [12].

Most previous devices that employ acoustic fields to manipulate microparticles create bulk standing waves through piezoelectric transducers. Recently, surface acoustic wave (SAW) devices have become more popular because of their design flexibility, ability for further downscaling, and on-chip integration through the use of interdigitated transducers (IDTs) [13]. Subtle positioning in 1-D or 2-D arrays with finer resolution down to the size of a single cell can be achieved by controlling the excitation frequency and configuration of the IDTs [14]-[19]. A sinusoidal pressure wave in the suspending medium is generated from the fluid-substrate interaction. One of the major challenges when interfacing the SAW substrate and the microfluidic chip is how to transmit the acoustic energy in desired locations inside the microchannel efficiently.

However, most polymeric materials commonly used for microfabrication, such as polydimethylsiloxane (PDMS) due to their low cost and ease of rapid prototyping, are unfortunately 


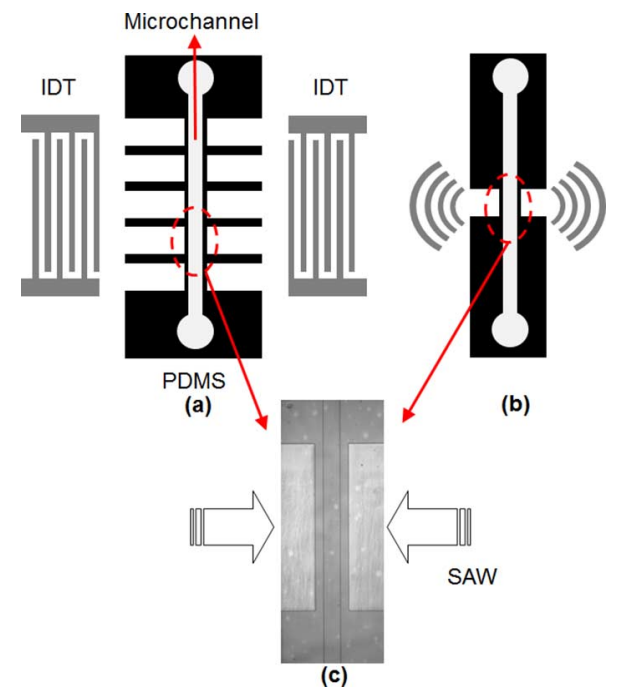

Fig. 1. Schematic illustration of the hollow PDMS-LiNbO ${ }_{3}$ chambers: the white regions represent the hollow chamber area, where the PDMS is not in contact with the substrate; the light grey region represents the microchannel; the black region represents the bulk PDMS in contact with the substrate; the dark grey region denotes the IDTs. (a) Chamber array with straight IDTs. (b) Pair of chambers with elliptical IDTs. (c) Microscopic image showing a pair of hollow chambers close to a microchannel (center, filled with liquid).

soft, and hence, acoustically very lossy [20]. Therefore, we used a popular design of hollow chambers formed by the PDMS channel and lithium niobate $\left(\mathrm{LiNbO}_{3}\right)$ substrate (Fig. 1) for interfacing of the SAW transducer with the microchannel. There is an air gap of $25 \mu \mathrm{m}$ in the chamber to avoid direct contact between the soft polymer and piezoelectric substrate, thereby reducing the acoustic energy absorption into PDMS. This design reduces the acoustic barrier to discrete PDMS channel walls, which has a minimal thickness just sufficient for proper channel sealing. Using this device, we studied the dynamics of the particle aligning under acoustic radiation force inside a microchannel by theoretical formulation, which has been briefly reported earlier [21]. Herein, in this work, the theoretical model was further expanded (Section II), and more importantly, the acoustic vibration of the channel substrate was directly measured and the data were compared with the results by numerical simulation (Section III).

\section{THEORY AND METHOD}

Fig. 2 shows the generation of an SSAW using an array of IDTs photolithographically patterned on a single-crystal $\mathrm{LiNbO}_{3}$ substrate $\left(128^{\circ}\right.$-rotated, $\mathrm{Y}$-cut, $X$-propagating, Roditi Ltd.) with a resonance frequency of $f_{R}=19.65 \mathrm{MHz}$. The IDT arrays patterned on the 4-in (diameter) $\mathrm{LiNbO}_{3}$ wafer were diced into small chips each with a size of $5 \mathrm{~cm} \times 2.5 \mathrm{~cm}$. Upon excitation by a continuous sinusoidal RF signal of $19.65 \mathrm{MHz}$, the IDTs generate SSAWs along the surface of the $\mathrm{LiNbO}_{3}$ substrate. Considering the sound velocity on the substrate surface $c_{L}=3788 \mathrm{~m} / \mathrm{s}$ [22], the SSAW has a wavelength of $193 \mu \mathrm{m}\left(\lambda=c_{L} / f_{R}\right)$. To investigate the effect of particle size with respect to the aggregation time, mono-dispersed fluorescent and nonfluorescent polystyrene particles with different

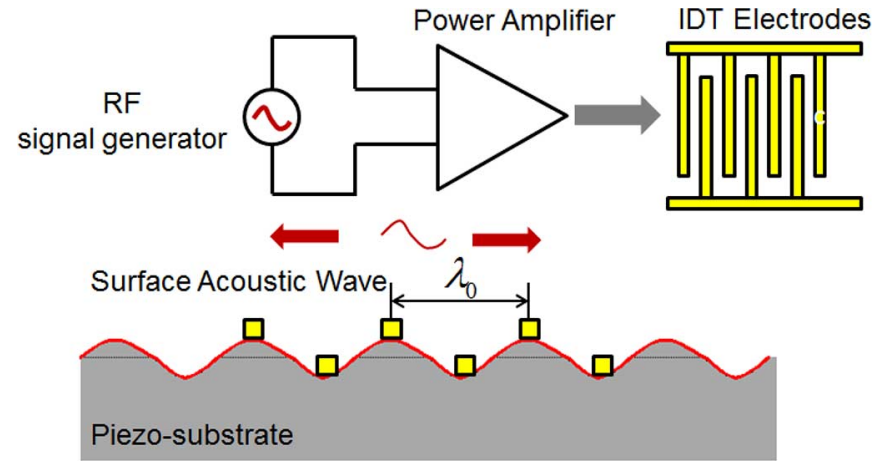

Fig. 2. Schematic illustration showing the generation of the SSAW using IDTs.

sizes were used in the experiment. The particle sizes used are $0.5,1,2.2,3,3.5,4.8$, and $5.7 \mu \mathrm{m}$, respectively. The particle suspension was introduced into the microchannel by a syringe pump. An inverted microscope and a charge coupled device (CCD) camera were used to visualize and record the particle aggregation.

\section{A. Dynamics of Particle Aggregation Under SSAW}

When a particle is suspended in liquid, it is subject to pressure fluctuations on its surface due to the longitudinal sound waves transmitted from the piezoelectric substrate to the liquid as the SSAW transverses it [23]. The pressure fluctuation culminates in an acoustic radiation force exerted on the particle, which consists of two major components due to both the rigid and compressible mechanical properties of the particle. Assuming the wavelength of the acoustic wave is $\lambda$, the wavenumber is $k=2 \pi / \lambda$, and the particle radius is $a$, we can introduce a characteristic parameter to indicate the aspect ratio between the particle size and the acoustic wavelength $k a$. For a small rigid sphere $(k a \ll 1)$ subject to a SSAW in the $x$-direction, Nyborg [24] developed a simple theoretical model that represents the rigid component of the acoustic radiation force

$$
F_{\text {rigid }}=V_{0}\left[\frac{3 \delta-3}{2 \delta+1}\left(\frac{\partial \bar{T}}{\partial x}\right)-\frac{\partial \bar{U}}{\partial x}\right]
$$

where $V_{0}$ is the volume of particle, $\delta=\rho_{p} / \rho_{f}$ is the density ratio between the particle and the suspending fluid, $\bar{T}$ is the time-averaged kinetic energy density, and $\bar{U}$ is the time-averaged potential energy density. The transient forms of $\bar{T}$ and $\bar{U}$ are given by

$$
T=\frac{1}{2} \rho_{f} u^{2}(x, t) \quad U=\frac{1}{2} \frac{p^{2}(x, t)}{\rho_{f} c_{f}^{2}}
$$

where $u$ is the velocity of the fluid material element, $p$ is the acoustic pressure, and $c_{f}$ is the sound speed in the fluid. For a planar standing wave, the acoustic pressure and material velocity along the $x$-direction are expressed as

$$
\begin{aligned}
& p(x, t)=P_{A} \cos (k x) \sin (\omega t) \\
& u(x, t)=\frac{P_{A}}{\rho_{f} c_{f}} \sin (k x) \cos (\omega t)
\end{aligned}
$$


where $P_{A}$ is the pressure magnitude. The time-averaged energy densities and their spatial gradient can then be expressed as

$$
\begin{aligned}
\bar{T} & =\frac{1}{T_{0}} \int_{-\frac{1}{2 T_{0}}}^{\frac{1}{2 T_{0}}} T d t=\frac{P_{A}^{2}}{4 \rho_{f} c_{f}^{2}} \frac{(1+\cos 2 k x)}{2} \\
\bar{U} & =\frac{1}{T_{0}} \int_{-\frac{1}{2 T_{0}}}^{\frac{1}{2 T_{0}}} U d t=\frac{P_{A}^{2} k}{4 \rho_{f} c_{f}^{2}} \frac{(1-\cos 2 k x)}{2} \\
\frac{\partial \bar{T}}{\partial x} & =-\frac{P_{A}^{2} k}{4 \rho_{f} c_{f}^{2}} \sin 2 k x \\
\frac{\partial \bar{U}}{\partial x} & =\frac{P_{A}^{2} k}{4 \rho_{f} c_{f}^{2}} \sin 2 k x .
\end{aligned}
$$

Substituting (5) into (1), the acoustic radiation force on a rigid sphere can then be obtained,

$$
F_{\text {rigid }}=-\frac{5 \delta-2}{2 \delta+1} \frac{V_{0} P_{A} k \sin 2 k x}{4 \rho_{f} c_{f}^{2}} .
$$

For a very small compressible spherical particle $(k a \ll 1)$, the time-averaged acoustic radiation force has been derived by Eller [25],

$$
F_{\text {compressible }}=-\left\langle V_{p}(t) \cdot \frac{\partial p(x, t)}{\partial x}\right\rangle
$$

where $V_{p}$ is the instantaneous particle volume. For a small sphere, $V_{p}$ can be written as [25]

$$
V_{p}(t)=V_{0}\left[1-P_{A} \frac{\sin (k x)}{\rho_{p} c_{p}^{2}} \cos \omega t\right]
$$

where $\rho_{p}$ and $c_{p}$ are the density and the sound speed in the particle material, respectively; $V_{0}$ is the original particle volume. Substituting (3) and (8) into (7), we can then obtain the instantaneous force attributed to the compressible component

$$
F_{\text {inst }}=-V_{0} k P_{A} \cos (k x) \cos \omega t+\frac{V_{0} k P_{A}^{2}}{2 \rho_{p} c_{p}^{2}} \sin (2 k x) \cos ^{2} \omega t .
$$

Integrating (9) over one wave cycle, the time-averaged force due to the compressible component reads

$$
F_{\text {compressible }}=\frac{k P_{A}^{2} V_{0} \sin (2 k x)}{4 \rho_{f} c_{f}^{2}}\left(\frac{1}{\delta \sigma^{2}}\right)
$$

where $\delta=\rho_{p} / \rho_{f}$ and $\sigma=c_{p} / c_{f}$.

Summing both the rigid and compressible contributions to the force in (6) and (10), the total acoustic radiation force acting on a particle is then

$$
F_{\text {acoustic }}=\frac{V_{0} P_{A}^{2} k \sin 2 k x}{4 \rho_{f} c_{f}^{2}}\left[\frac{5 \delta-2}{2 \delta+1}-\frac{1}{\delta \sigma^{2}}\right]
$$

where $\phi=(5 \delta-2) /(2 \delta+1)-(1) /\left(\delta \sigma^{2}\right)$ represents the acoustic contrast factor of the particle relative to the suspending fluid, and $\varepsilon=\left(P_{A}^{2}\right) /\left(4 \rho_{f} c_{f}^{2}\right)$ represents the acoustic energy input into suspending medium. Therefore, the total acoustic force in (11) can be simply written as

$$
F_{\text {acoustic }}=k \phi V_{0} \varepsilon \sin (2 k x) \text {. }
$$

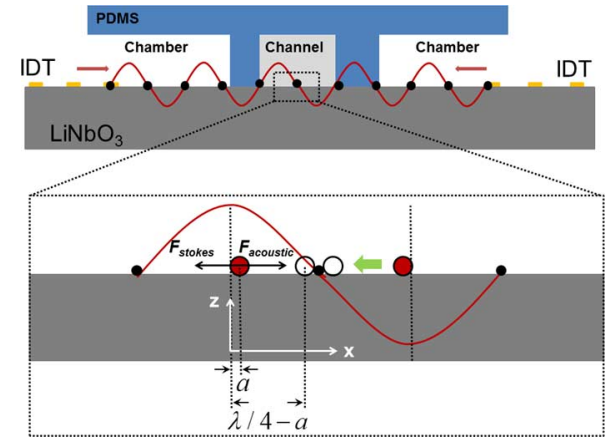

Fig. 3. Schematic illustration of the device and working principle of the SSAW particle focusing in a microfluidic channel.

The Stokes drag force on a spherical particle moving in fluid, on the other hand, is expressed as [23]

$$
F_{\text {stokes }}=6 \pi \mu a u_{p}
$$

where $\mu$ is the fluid viscosity, and $u_{p}$ is the velocity of the particle relative to the fluid. Here, we only consider the particle focusing under moderate acoustic power, which is sufficiently low such that acoustic streaming is negligible; therefore, in the absence of fluid flow, the relative particle velocity is the same as its absolute velocity.

The particle motion involves two phases, as illustrated in Fig. 3: an initial acceleration transient followed by an equilibrium phase. When the acoustic field is first applied, the particle starts to move under the acoustic radiation force. Since $F_{\text {stokes }}$ is proportional to the particle velocity, the initial frictional force is smaller than $F_{\text {acoustic }}$ when the velocity is still low, and hence the particle accelerates. After a short moment when the particle velocity reaches a threshold value, $F_{\text {stokes }}$ becomes equal to $F_{\text {acoustic }}$, and the particle reaches an equilibrium state and moves at a constant velocity. The dynamic equation of the particle motion is expressed as

$$
F_{\text {acoustic }}-F_{\text {stokes }}=m_{p} \frac{d u_{p}}{d t} .
$$

Substitute (13) into (14) and solve the differential equation, we obtain the particle velocity

$$
u_{p}=\frac{F_{\text {acoustic }}}{6 \pi \mu a}\left[1-\exp \left(-\frac{6 \pi \mu a}{m_{p}} t\right)\right] .
$$

The characteristic time scale for the particle to reach steady state is then

$$
\tau_{p}=\frac{m_{p}}{6 \pi \mu a} \sim O\left(\frac{a^{2} \rho_{p}}{\mu}\right) .
$$

Considering a particle of radius $a=10 \mu \mathrm{m}$ and density of $\rho=1000 \mathrm{~kg} \mathrm{~m}^{-3}$, the characteristic time is approximately 0.1 $\mathrm{ms}$. Since this acceleration phase is extremely short compared to the time scale over which the external forces vary, the acceleration phase is negligible, and hence the particle appears to move at an equilibrium velocity expressed as

$$
u_{p}=\frac{F_{\text {acoustic }}}{6 \pi \mu a} .
$$


Substitute (12) into (14d) and consider the original particle volume $V_{0}=4 \pi a^{3} / 3$, the equilibrium velocity reads

$$
u_{p}=\frac{\lambda(k a)^{2}}{9 \pi \mu} \phi \varepsilon \sin 2 k x
$$

where $\lambda=2 \pi / k$ is the acoustic wavelength. Since $u_{p}=d x / d t$, integrating (15) then allows the maximum time required for the particle to assemble at the pressure nodes to be derived,

$$
t=\int_{0}^{t} d t=\int_{a}^{\frac{\lambda}{4}-a} \frac{1}{u} d x=\frac{9 \pi \mu}{\phi \varepsilon} f
$$

where $f$ is a time scale factor given by

$$
f=\frac{\ln [\cot (k a)]}{2 \pi(k a)^{2}} .
$$

Obviously this factor is only dependent on the aspect ratio of the particle $k a$. As implied by (16), the total time taken for a particle to aggregate at the pressure node is proportional to the time scale factor $f$ for a given power input and acoustic contrast factor. The integration limit in (16) for the particle displacement $x$ is from $(\lambda / 4-a)$ to $a$, as shown in Fig. 3. This mathematical manipulation avoids the problem that, theoretically, the particle can maintain equilibrium both at the antinodes (unstable equilibrium due to the acoustic pressure balance) and nodes (stable equilibrium due to zero acoustic pressure). In reality, however, the particles initially located at antinodes can be easily deflected by weak disturbances and move to the nodes eventually.

\section{B. Numerical Modeling of SSAW}

To simulate the generation of SSAW in this system, we established a simplified 2-D model using a finite-element method (FEM) commercial package (COMSOL Multiphysics 4.3b) in which the acoustic-piezoelectric interaction module in the frequency domain was selected for multi-physical coupling. This acoustic-piezoelectric system can be generally described by the Maxwell's equations and the stress-strain equations [27]

$$
\begin{aligned}
T_{i j} & =C_{i j k l}^{E} \cdot S_{k l}-e_{i j k}^{T} \cdot E_{k} \\
D_{i} & =e_{i k l} \cdot S_{k l}+\varepsilon_{i j}^{S} \cdot E_{j}
\end{aligned}
$$

where $T$ is the mechanical stress vector, $C^{E}$ is the elasticity matrix, $S$ is the strain vector, $e$ is the piezoelectric stress matrix, $E$ is the electric field vector, $D$ is the electric displacement vector, and $\varepsilon$ is the dielectric matrix. The superscript " $T$ " indicates the transpose of the matrix.

For simplicity, the computation domain is limited to a thin region of the piezoelectric substrate underneath the IDTs since the mechanical motion is limited near the surface. A sinusoidal electric voltage with a peak-to-peak magnitude of $0.35 \mathrm{~V}$ and frequency of $19.65 \mathrm{MHz}$ is applied as the electrical boundary condition at the IDT-substrate interface, while other boundaries of the piezoelectric substrate were assumed to be zero charge. Mechanically, the boundary of IDTs was set as free surface since there is no applied load or constraint. An adaptive mesh setting was applied in this model, in which fine mesh size was used for most computation domain, while ultra-fine mesh size was specified for the region near the electrodes. Generally, the mesh size in this model has a maximum of $2 \mu \mathrm{m}$ and a minimum of

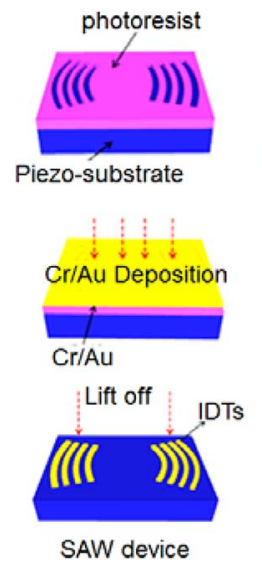

(a)
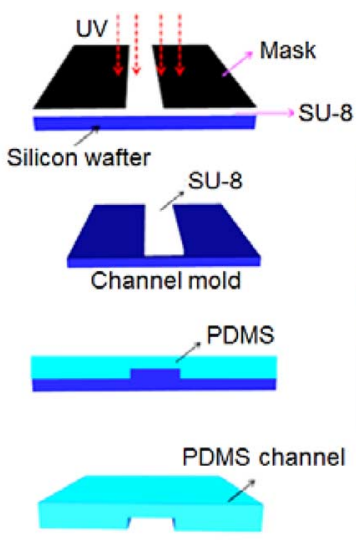

(b)

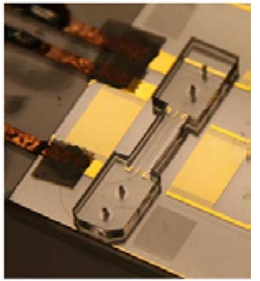

Fig. 4. Fabrication procedure of the: (a) SSAW device and (b) microfluidic channel. (c) Final assembled device ready for use.

$0.1 \mu \mathrm{m}$. The entire unknown elements generated in the meshing are 1.09 million.

\section{Device Fabrication}

In order to fabricate the IDTs, an $\mathrm{LiNbO}_{3}$ substrate was first patterned with a thin layer of photoresist with the desired negative IDT structure using photolithography. The patterned substrate was then deposited with dual metal layers (Al/Ti) to enhance the bonding of the electrodes with $25-\mu \mathrm{m}$-thick aluminum sputtered on top of a 4-nm titanium adhesion layer. After a lift-off process, the metal IDTs were created on the substrate with a finger and gap width of $49 \mu \mathrm{m}$. A PDMS microchannel with hollow side chambers was then fabricated by standard soft lithography. The microchannel has a width of $290 \mu \mathrm{m}$, height of $25 \mu \mathrm{m}$, and length of $2 \mathrm{~cm}$. Finally, the PDMS channel and the $\mathrm{LiNbO}_{3}$ substrate were aligned and bonded. The detailed fabrication procedures are illustrated in Fig. 4.

\section{RESULTS AND DISCUSSION}

The SSAW can be characterized by monitoring the displacement of the substrate material along the surface. Fig. 5(a) shows the simulated material displacement along the $\mathrm{LiNbO}_{3}$ substrate at the IDT region. Fig. 5(b), ${ }^{1}$ on the other hand, shows the measured surface displacement of the oscillation on the substrate beneath the microfluidic channel using a scanning laser Doppler vibrometer (LDV) system. Both simulated results and experimental results indicate that the sinusoidal SSAW has a wavelength of $190 \pm 5 \mu \mathrm{m}$, which is consistent with the theoretical analysis in the previous section. However, the simulated magnitude of surface displacement [about $0.25 \mathrm{~nm}$ in Fig. 5(a)] is greater than the measured magnitude $[0.12 \pm 0.02 \mathrm{~nm}$ in Fig. 5(b)]. We speculate that this discrepancy is mainly due to the attenuation when the SSAW propagates from the IDTs to the channel, and also due to the energy dissipation from the substrate to the loading fluid inside the channel.

As an important objective of this study, we investigated the dynamics of particle aggregation along the acoustic pressure nodal lines in a microchannel. Fig. 6 shows the chronological

\footnotetext{
${ }^{1}$ See also the supplementary video available on Xplore
} 

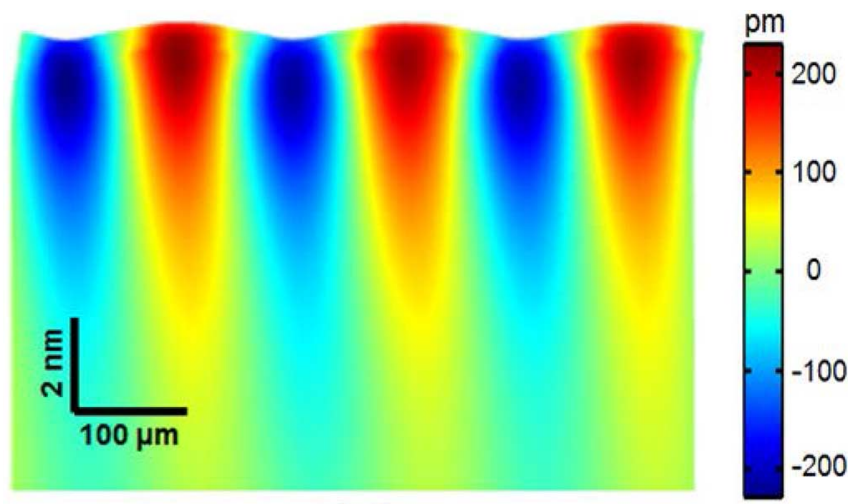

(a)

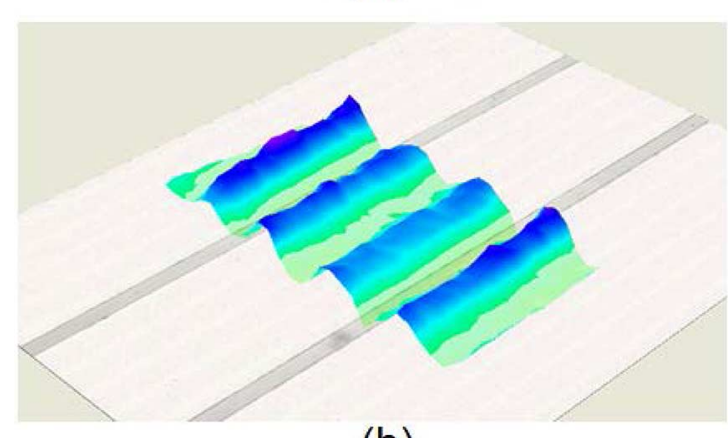

(b)

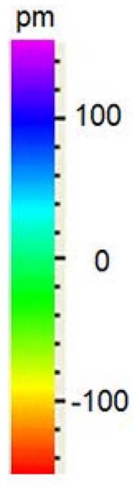

Fig. 5. (a) Simulated material displacement along the surface of $\mathrm{LiNbO}_{3}$ substrate. (b) Transient displacement on the substrate surface beneath the microchannel measured by a micro-scanning LDV system. Two grey bands on the substrate surface represent the location of the PDMS microfluidic channel walls.
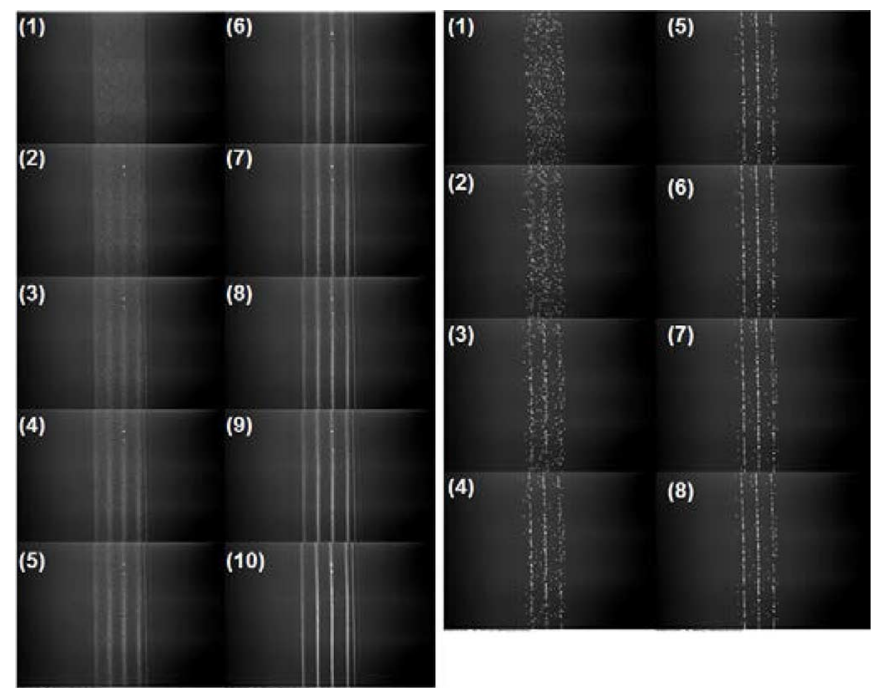

Fig. 6. Time-lapse images of particle alignment in a microfluidic channel under SSAW. The left panel shows the alignment dynamics of $0.5-\mu \mathrm{m}$ particles over the first $5.6 \mathrm{~s}$ (time interval $0.62 \mathrm{~s}$ ). The right panel shows the alignment dynamics of $2.2-\mu \mathrm{m}$ particles, with total time taken of $2.8 \mathrm{~s}$ (time interval of $0.4 \mathrm{~s}$ ). The numbering indicates the time sequence of the images.

image sequence of particle aggregation under the SSAW, using 0.5 - and $2.2-\mu \mathrm{m}$ particles as examples. Upon excitation by a sinusoidal RF signal with the same frequency as the IDT resonance frequency, the SSAW exerts acoustic radiation force on

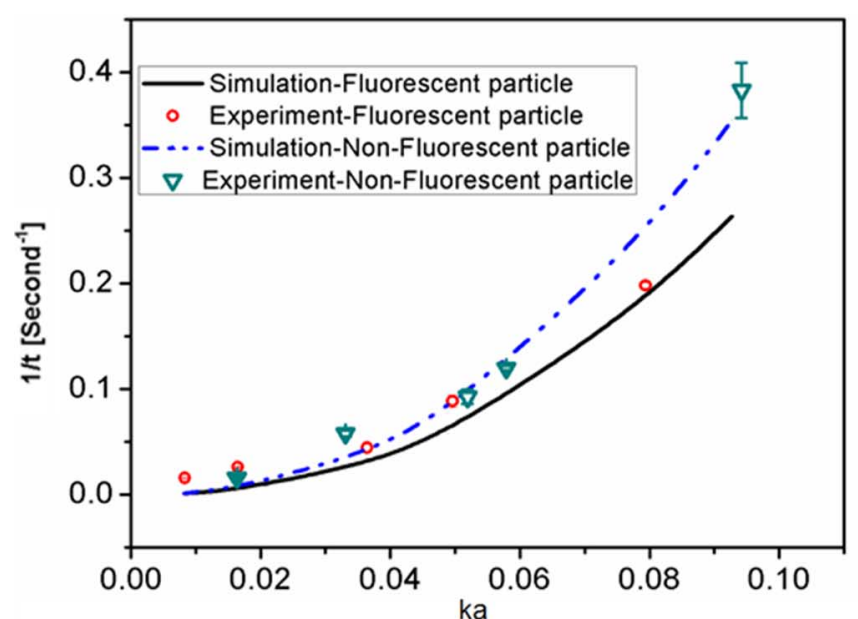

(a)

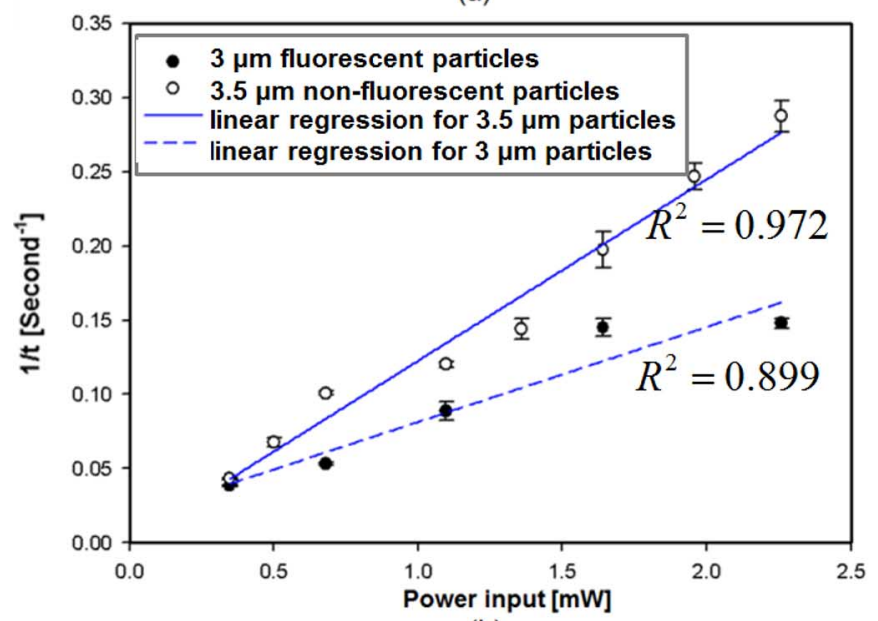

(b)

Fig. 7. (a) Reciprocal of the aggregation time $(1 / t)$ as a function of the particle aspect ratio $k a$. (b) Reciprocal of the aggregation time as a function of power input. The dashed and solid lines in (b) represent the linear regression fit of the data.

the microparticles, which are pushed to the pressure nodal lines where they are subject to zero net force. In Fig. 6, both microparticles are homogeneously dispersed in the carrying liquid initially (image \#1). After excitation of the RF-activated SSAW, all the particles start to accumulate rapidly along several straight lines in the microchannel. Using the scanning LDV measurement [see Fig. 5(b)], we have verified that these straight lines are corresponding to the acoustic pressure nodes inside the microchannel. Obviously it takes much longer time for $0.5-\mu \mathrm{m}$ particles to aggregate compared to $2.2-\mu \mathrm{m}$ particles.

As implied in (16), the time taken for this induced aggregation process depends on the ratio of particle size relative to the SSAW wavelength $k a$, the acoustic contrast factor $\phi$ of the particle relative to the suspending medium, and the acoustic power input into the carrying liquid. Therefore, we studied the effects of the particle size and acoustic power on the aggregation time.

The experimental results of the aggregation time for particles with various sizes, both fluorescent and nonfluorescent, are shown in Fig. 7(a). It should be noted that it is rather challenging to determine the accurate aggregation time of extremely small particles (close to or below $1 \mu \mathrm{m}$ ) due to strong diffusion 
that is working against further acoustic focusing after aggregation occurs. It has been observed that when the RF excitation is switched off, the aggregated particles, especially for particles smaller than $1 \mu \mathrm{m}$, disperse away from the nodal lines, while the particles larger than $2 \mu \mathrm{m}$ can still maintain their alignment along the nodal lines.

In order to tone down the measurement error due to this uncertainty, the reciprocal of aggregation time $(1 / t)$ is presented as a function of the aspect ratio $(k a)$, according to the following equation:

$$
\frac{1}{t}=\frac{\phi \varepsilon}{9 \pi \mu} \frac{1}{f} .
$$

The theoretical analysis in this work predicts that, for a given acoustic power and SSAW wavelength, the aggregation time decreases with increasing the particle size. The experimental data in Fig. 7(a) show the major trend as predicted by the theory, although the nonfluorescent and fluorescent particles follow slightly different trends. We speculate that this deviation may arise from the difference in the acoustic contrast between these two groups of particles.

The particle aggregation under different acoustic power was also studied for $3-\mu \mathrm{m}$ fluorescent particles and $3.5-\mu \mathrm{m}$ nonfluorescent particles. The experimental results in Fig. 7(b) show that the aggregation time is inversely proportional to the power input, as predicted by (20). This result implies that relatively higher power input is desirable for rapid particle aggregation. However, in reality, one should also consider other side effects under extremely high acoustic power, such as acoustic streaming, heating, and bubbling due to the liquid evaporation. Fortunately, it is found that the microfluidic flow in this SSAW device is free of acoustic streaming or heating under moderate power input in the range of a few milliwatts.

\section{CONCLUSION}

This study has mainly focused on the analysis of the particle focusing dynamics using an SSAW in a microfluidic channel under different effects, such as particle materials, particle size, and acoustic power. The analytical model was derived to predict the focusing time to evaluate how fast the acoustic focusing can be realized, which has not been shown in prior studies [27]. In addition, as another major contribution, the instantaneous mechanical vibrations inside this acoustofluidic system were directly measured with a micro-scanning LDV, and the experimental data showed acceptable agreement with the numerical simulation. The time taken for the particle to align along the acoustic pressure nodes was observed to depend on the acoustic power of the vibration, the acoustic contrast of the particle relative to the suspending medium, and the aspect ratio of the particle size to the acoustic wavelength. Specifically, the particle aggregation time decreases if the acoustic power increases, or if the particle has greater size or acoustic contrast factors.

\section{REFERENCES}

[1] M. Toner and D. Irimia, "Blood-on-a-chip," Annu. Rev. Biomed. Eng., vol. 7, pp. 77-103, 2005.
[2] J. El-Ali, P. K. Sorger, and K. F. Jensen, "Cells on chips," Nature, vol. 442, pp. 403-411, 2006.

[3] C. D. Chin, V. Linder, and S. K. Sia, "Lab-on-a-chip devices for global health: Past studies and future opportunities," Lab. Chip., vol. 7, no. 1, pp. 41-57, 2007.

[4] D. V. Lim, J. M. Simpson, E. A. Kearns, and M. F. Kramer, "Current and developing technologies for monitoring agents of bioterrorism and biowarfare," Clin. Microbiol. Rev., vol. 18, no. 4, pp. 583-607, 2005.

[5] R. H. Liu, J. N. Yang, R. Lenigk, J. Bonanno, and P. Grodzinski, "Selfcontained, fully integrated biochip for sample preparation, polymerase chain reaction amplification, and DNA microarray detection," Anal. Chem., vol. 76, no. 7, pp. 1824-1831, 2004.

[6] F. Guo, X. Ji, K. Liu, R. He, L. Zhao, Z. Guo, W. Liu, S. Guo, and X. Zhao, "Droplet electric separator microfluidic device for cell sorting," Appl. Phys. Lett., vol. 96, 2010, Art. ID 193701.

[7] X. Wang, S. Chen, M. Kong, Z. Wang, K. D. Costa, R. A. Li, and D. Sun, "Enhanced cell sorting and manipulation with combined optical tweezer and microfluidic chip technologies," Lab. Chip., vol. 11, no. 21, pp. 3656-3662, 2011.

[8] J. Loureiro, P. Z. Andrade, S. Cardoso, C. L. da Silva, J. M. Cabral, and P. P. Freitas, "Magnetoresistive chip cytometer," Lab. Chip., vol. 11, no. 13, pp. 2255-2261, 2011.

[9] M. Wiklund and H. Hertz, "Ultrasonic enhancement of bead-based bioaffinity assays,” Lab. Chip., vol. 6, no. 10, pp. 1279-1292, 2006.

[10] L. A. Kuznetsova and W. T. Coakley, "Applications of ultrasound streaming and radiation force in biosensors," Biosens. Bioelectron., vol. 22, no. 8, pp. 1567-1577, 2007.

[11] A. Lenshof, M. Evander, T. Laurell, and J. Nilsson, "Acoustofluidics 5: Building microfluidic acoustic resonators," Lab. Chip., vol. 12, no. 4, pp. 684-695, 2012.

[12] G. Goddard and G. Kaduchak, "Ultrasonic particle concentration in a line-driven cylindrical tube," J. Acoust. Soc. Amer., vol. 117, no. 6, pp. 3440-3447, 2005.

[13] L. Y. Yeo and J. R. Friend, "Ultrafast microfluidics using surface acoustic waves," Biomicrofluid., vol. 3, no. 1, 2009, Art. ID 012002.

[14] C. D. Wood, S. D. Evans, J. E. Cunningham, R. O'Rorke, C. Wälti, and A. G. Davies, "Alignment of particles in microfluidic systems using standing surface acoustic waves," Appl. Phys. Lett., vol. 92, no. 4, 2008, Art. ID 044104.

[15] C. D. Wood, J. E. Cunningham, R. O'Rorke, C. Wälti, E. H. Linfield, A. G. Davies, and S. D. Evans, "Formation and manipulation of twodimensional arrays of micron-scale particles in microfluidic systems by surface acoustic waves," Appl. Phys. Lett., vol. 94, no. 5, 2009, Art. ID 054101.

[16] J. Shi, X. Mao, D. Ahmed, A. Colletti, and T. J. Huang, "Focusing microparticles in a microfluidic channel with standing surface acoustic waves (SSAW)," Lab. Chip., vol. 8, no. 2, pp. 221-223, 2008.

[17] J. Shi, D. Ahmed, X. Mao, S. C. S. Lin, A. Lawit, and T. J. Huang, "Acoustic tweezers: Patterning cells and microparticles using standing surface acoustic waves (SSAW)," Lab. Chip., vol. 9, no. 20, pp. 2890-2895, 2009.

[18] L. Meng, F. Cai, Z. Zhang, L. Niu, Q. Jin, F. Yan, J. Wu, Z. Wang, and H. Zheng, "Transportation of single cell and microbubbles by phase-shift introduced to standing leaky surface acoustic waves," Biomicrofluid., vol. 5, no. 4, 2011, Art. ID 044104.

[19] N. D. Orloff, J. R. Dennis, M. Cecchini, E. Schonbrun, E. Rocas, Y Wang, D. Novotny, R. W. Simmonds, J. Moreland, I. Takeuchi, and J. C. Booth, "Manipulating particle trajectories with phase-control in surface acoustic wave microfluidics," Biomicrofluid., vol. 5, no. 4, 2011, Art. ID 044017.

[20] S. M. Langelier, L. Y. Yeo, and J. Friend, "UV epoxy bonding for enhanced SAW transmission and microscale acoustofluidic integration," Lab. Chip., vol. 12, pp. 2970-2976, 2012.

[21] J. Guo, Y. Chen, and Y. Kang, "RF-activated surface standing acoustic wave for on-chip controllably aligning of bio-microparticles," in IEEE MTT-S Int. Microw. Workshop RF Wireless Technol. Biomed. Healthcare Appl. Series, Dec. 9-11, 2013, pp. 1-3.

[22] H. Li, J. R. Friend, and L. Y. Yeo, "Microfluidic colloidal island formation and erasure induced by surface acoustic wave," Phys. Rev. Lett., vol. 101, 2008, Art. ID 084502.

[23] J. R. Friend and L. Y. Yeo, "Microscale acoustofluidics: Microfluidics driven via acoustics and ultrasonics," Rev. Mod. Phys., vol. 83, pp. 647-704, 2011.

[24] W. L. Nyborg, "Self-maintained oscillations of the jet in the jet-edge system," J. Acoust. Soc. Amer., vol. 26, pp. 174-182, 1954.

[25] A. Eller, "Force on a bubble in a standing acoustic wave," J. Acoust. Soc Amer., vol. 43, pp. 170-171, 1968. 
[26] L. A. Crum, "The acoustic radiation pressure on a liquid droplet in a stationary sound field," Michelson Phys. Lab., U.S. Naval Academy, Annapolis, MD, USA, Tech. Rep. No. C-1, 1970.

[27] Y. Ai, C. K. Sanders, and B. L. Marrone, "Separation of E. coli bacteria from peripheral blood mononuclear cells using standing surface acoustic waves," Anal. Chem., vol. 85, no. 19, pp. 9126-9134, 2013.

Jinhong Guo (M'14), photograph and biography not available at time of publication.

James R. Friend, photograph and biography not available at time of publica-

Yuejun Kang, photograph and biography not available at time of publication. publication. tion.

Yu Chen, photograph and biography not available at time of publication.

Leslie Y. Yeo, photograph and biography not available at time of publication. 